Katsue Akiba Reynolds

\title{
Female Speakers of Japanese
}

A woman has no master. She must consider her husband her master and serve him with respect. Generally, the way of a woman lies in obedience. Towards her husband, she must be polite, subservient, and humble in her expression and language. She must neither be impatient or disobedient. nor proud or rude. This is the foremost obligation of a woman.

- Onna Daigaka

Spurred by the women 's movement, the issue of sex differentiation has recently been given more attention in more areas of study than it has at any time in the past. Linguistics is one of the areas that cannot avoid commitment to the trend. Volumes by Key (1975) and Lakoff (1975) and articles collected in Thorne and Henry (1975) and Orasanu. Slater, and Adler (1979) are among the numerous contributions on the topic of sex differentiation in languages during the past decade.

One of the major concerns of these linguists is the difference between female and male speech and the possible reasons for it. All agree that the linguistic discrepancy reflects social inequity between men and women: woman's language "submerges a woman's personal identity, by denying her the means of expressing herself strongly, on the one hand, and encouraging expressions that suggest triviality in subject matter and uncertainty about it" (Lakoff 1975:7). There are a number of interesting linguistic phenomena to be persuasively accounted for on this view. One of the prominent examples concerns the fact that English-speaking women tend to use rising intonation in the context where men would use falling intonation. Given that rising intonation expresses the speaker's uncertain psychological state about the content of the statement and that it is typical of question sentences, the use of rising intonation in declarative sentences by women is explained as follows: women are dominated socially by men and are generally less secure 
about themselves. At the same time culture imposes an image of women as "fragile" and unable to assert themselves. As a resuli, women speakers tend, consciously or not, to seek to conform to the image, using rising intonation even in declarative sentences, as if "seeking confirmation" (Lakoff 1975:17).

Most of the studies on the question of sex differentiation in language assume, explicitly or implicitly, that the differentiation exists at all levels of grammar and that the correlation between women's language and inferior social status is universal. The data so far presented, however, are not sufficient for such claims. There have been too few syntactic examples, and most examples from languages other than English are fragmentary. Japanese, a language in which male and female speech are exclusively differentiated to a significant degree and spoken in a society characterized by overt social discrimination between men and women, provides an excellent case for further exploration of this issue.

In the present paper, I shall focus on "final mode," a feature peculiar to Japanese, and will attempt to show that women. having no access to superior-inferior modes, can talk with impunity only as inferiors, i.e., with less assertion and more formality and/or politeness. ${ }^{2}$ Then, I would like to call attention to a sociopsychological problem that Japanese women may be confronted with as a result of the conflict between this linguistic disparity and recent trends in Japan towards more equality. Finally I shall suggest that sex differentiation in Japanese will eventually be minimized.

\section{Final Particles}

According to Nakane (1970), the Japanese are a rank-conscious people. In characterizing Japanese society as "vertical," as opposed to a "horizontal" society such as American society she suggests that socjal groups in Japan have or tend to have an elaborately hierarchical internal structure by which the members are tied vertically into a delicately graded order. She states, "Without consciousness of ranking, life could not be carried on smoothly in Japan" (Nakane 1970:31). Linguistic activity is no exception to this principle of verticality. When speaking, one always defines his/her rank relative to the hearer's and chooses the most appropriate level of speech. In other words, the speaker must indicate in his/her language as well as in his/her manner the rank relationship that he or she perceived. The appropriateness in this process is particularly important when the speaker is socially inferior to the hearer.

There are two major linguistic categories that are especially relevant for this differentiation of speech level: addressing and referring terms and what 
may be called "final mode." The differentiation made by forms of addressing is analogous to the distinction between $t u$ and $v o u s$ in French except that the gradation in usage is far more elaborate in Japanese. Takao Suzuki (1972) proposes an interesting analysis of terms referring to speakers themselves in Japanese and the Japanese speakers' psychology underlying it. He points to the fact that Japanese pronouns such as watashi, I, and anata, you, are not as commonly used as Indo-European pronouns and that lexical nouns indicating the speaker's position vis-à-vis the hearer, are used instead. For example, if the speaker is a teacher or an uncle of the hearer, the speaker as well as the hearer would refer to the speaker himself by Sensei, teacher, or Oji-san, uncle. Suzuki attributes this to the Japanese speaker's mentality. In speech situations a Japanese speaker defines himself or herself in terms of the specific relationship between himself or herself and the hearer in more detail than simply as the speaker. Suzuki explains the fact that a Japanese often remains silent in English-speaking interactions with foreigners as follows: we Japanese can define ourselves only in relation to specific hearers and can determine our attitudes and behavioral patterns ("ego-reification"), while Westerners always define themselves as "I" when their role in interactions is that of the speaker, and as "you" when it is that of the hearer ("ego-modification"). Facing foreigners who don't fit in any relational pattern within Japanese culture, Japanese speakers become totally disoriented and feel extremely uncertain about themselves. As a result we are not able to participate in interactions. To be able to place the speaker and other participants properly within the conventional social frame and to be able to choose proper addressing and referring terms are a prerequisite for Japanese speakers to comfortably participate in conversational situations. In this study, however, I will not deal directly with differentiation in addressing/referring terms but will focus on final mode, a category which I believe reveals more interesting facts about sex differentiation in language.

Every Japanese sentence ending may be said to express the speaker's attitude towards the hearer. If the speaker is ranked as superior to the hearer, he or she will be inclined to end the sentence with a more dominant mode than the hearer might use, and vice versa. How the ranks are determined in each relationship is a big question to be answered in an independent study but it is certain that sex and age are among the major variables. If women are generally considered to be inferior, the difference in final mode between male and female speech should correlate to some degree with the social difference, and it should tell us more specifically how men and women differ in their social behavior.

Final mode dominance is a function of the degree of assertion of the 
final particle (FP), formality, intonation, and the explicitness of mood, such as declarative, imperative, and interrogative. The interaction between these properties is so intricate that discussing them separately is almost impossible. In this first section, I shall deal with final particles; in the later sections, I shall focus on syntactic rules involved in declarative and imperative constructions and on formal speech. Intonation will be discussed wherever it is relevant.

\section{Ranking and Final Particles}

As a point of departure, let us compare the following two sentences. Differing only in final mode. they convey the same message. "Masao talked to Kazuko (about it)"

(Abbreviations used in the sample sentences are as follows:

SUBJ: Subject Marking Particle placed after the subject nominal.

DO: Direct Object Marking Particle placed after the direct object nominal.

TOP: Topic Marking Particle placed after the topic nominal.

+IMP: Imperative Form.

+FML: Formal Form.

FML: Formal Verbal Suffix.

PAST: Past Tense Marker.

COP: Copula.

NOM: Nominalizer.

FP: Sentence Final Particle.

Also, I used "\&" to indicate the conjunctive particle te that occurs between two verbs in a serial construction, and 1 glossed the second verb with its literal meaning written in capital letters, e.g., GIVE in 17, 18, and 19.)
(1)-(a) Masaoga Kaztko ni hanashi-ta
Wasao SUBJ Kazuko to talk- PAST
(b) Masanga Kazakeni hanashi-ta vo.
Masao SUBJ Kazuke to talk- PAST FP

The final particle $w$ stands in no grammatical relation to the rest of the sentence. Nor does it change the mood of the sentence: both (a) and (b) are unequivocally declarative sentences. In addition to $y \theta$, there are at least four other particles used primarily in declarative sentences: $z o, z e, s a$, and wa (if we limit ourselves to so-called standard Japanese). The use of these varia- 
tions is largely based on the rank relationship between the speaker and the hearer, and therefore it is one of the most difficult aspects of the language for foreigners to master.

The difference between the sentence with FP and those without (which we may abbreviate as [S] and [S-FP] respectively), although not immediately apparent, emerges when placed in discourse perspective. The [S] pattern is characteristically used in written discourse, and the [S-FP] pattern exclusively in conversation. Although the [S] pattern is occasionally used in conversation, it sounds too brusque or gives the impression that the speaker is indifferent to the hearer's viewpoint. It is almost prohibitive in women's specch unless the predicate is formal.

Written discourse and conversational discourse are highly contrastive with respect to the potential of the addressee's immediate response. In the former, the writer and the reader are spatially separated, coding and decoding time are not simultaneous, and the writer has no specific reader in mind (special cases of writing, like letters, are exceptions to this). And consequently, the reader cannot be expected to participate in the speech act which the writer is performing. In conversation, on the other hand, there is a specific individual with the role of the hearer located within the immediate space of the speaker (this aspect is only psychological in phone conversation) and the speaker is aware of the potential of the hearer's immediate reaction. ${ }^{3}$

The basic function of FP is to acknowledge this potential and thereby to create a conversational situation or maintain this situation that has already been created. The fact that Japanese has this peculiar category of FP may be taken as another piece of evidence in support of Suzuki's hypothesis (op.cit.) about the Japanese pattern of ego-reification.

Final particles are complexes of subtle semantic and/or speech act features and are difficult to define in precise terms at the present stage of knowledge. One feature of particles that can be discussed with some confidence is their relative degree of assertion, which can be indirectly determined by using the superior-inferior relation as a framework, assuming that the superior is allowed to be more assertive than the inferior but not vice versa. The following observation by Nakane illustrates well how the rank awareness of the Japanese dictates some of the rules of conversation in Japanese:

The consciousness of rank which leads the Japanese to ignore logical procedure is also manifested in the patterns and practices of daily conversation, in which a senior or an elderly man monopolizes the talk while those junior to them have the role of listener. Generally there is no development of dialectic style in 
Japanese conversation, which is guided from beginning to end by the interpersonal relations which exist between the speakers. (Nakane 1970:34)

This is exactly the rule that Brown and Gilman (1968) discuss as "the power semantic." The power semantic is nonreciprocal; the superior uses $T$ (informal second person pronoun $t u$, in French for example) and receives $\mathrm{V}$ (French vous). It is always the superior who initiates, changes the direction of conversation and generally controls the situation by asserting his own standpoint. Violation of the rule, especially by the inferior, provokes special attention and invites special inference of the intention.

It is reasonable to assume that a particle that can be used only by the superior has more assertive force than one that the inferior can use. For the purpose of keeping at least the variable of sex constant, the rank relationships first to be referred to in considering the degree of assertion of FP will be limited to those held among males.

\section{Declaratives}

Impressionistically, the FP that sounds most dominant is $z o$. The $\mathrm{S}-z o$ pattern is appropriate only when the speaker is superior or equal to the hearer in social ranking, e.g., father-son, employer-employee, teacher-student, senior student-junior student. Zo has such a strong overtone that it accompanies sentences of assertive illocutionary act types such as revealing, warning, and pointing out, and it occurs with sentences initiating conversational situations rather than in sentences responding to something already said. The following sentence from a contemporary novel ${ }^{4}$ is uttered by a company employee, the hero of the plot, to a gatekeeper upon entering the clearance station:

(2) Oi, kat- te-ki- la zo:

Hey return- \&-come- PAST FP

Hey, I'm back!

$Z e$ is almost equal to zo in its degree of assertion but it is less authoritative. ${ }^{5}$ It is used only towards the speaker's equal. It is not appropriate in an inferior-superior situation, however small the gap between the two ranks may be. The fact that $z e$, not $z 0$, can occur in a hortative sentence may also be an indication that its assertion is slightly milder than that of $z o$.

The assertive force of $s a$ is somewhat different in nature. It is typically used in responding to the conversational situation that has already been 
initiated by the other speaker. However, the response is not the I-am-readyto-answer-your-question or I-agree-with-you type, but it indicates the speaker's defiance of the situation. ${ }^{6}$ To respond with Dooshite sa, "Why?", to a question, "Do you speak English?", for example, apparently shows unwillingness to cooperate with the interrogator. The attitude expressed by $s a$ is defensive but not passive: it indicates the speaker's intent not to be dominated by the other. It is used only toward equals. It is interesting to note that young women today use sa more frequently and in more public places than before, although its use is limited to the interactions among themselves and is generally frowned on by society.

$Y_{0}$, the most widely used declarative particle, is certainly assertive, but it is only slightly more assertive than the declarative sentence would necessarily be. It can be used in many inferior-superior situations, such as sonfather, junior student-senior student, etc., if the relationship is of an intimate kind. ${ }^{7}$ The feature of $y o$ that is more distinctive than assertiveness may be the potential of the hearer's immediate response. It is more often than not pronounced with a fall-rise intonation.

Occurring with hortative or imperative sentences, yo seems to soften the dominating tone inherent in those sentences. In contrast to $I k o 0$, "Let's go," which has a strong sense of determination, Ikoo yo sounds as if the speaker is asking for the hearer's consent and may be best translated into English as "Let's go, shall we?"

Wa distinguishes itself from the other declarative particles by the weakness of the speaker's intention to involve the hearer in the speech act. By uttering [ $[S]-w a]$, the speaker neither imposes his/her standpoint nor invokes the hearer's opinion. ${ }^{8}$ The [IS]-wa] pattern therefore cannot perform a speech act which requires a more or less specific verbal or nonverbal response (request and command). It never occurs in an imperative or hortative construction. It appears most commonly with sentences stating the speaker's own state of mind or intention (e.g., Komat-ta wa. "I am in trouble," and Boku iku wa, "I will go") or with those describing a situation obvious to both speaker and hearer (e.g.. Aitsu mata nonde-nu wa, "That guy, he is drinking again!" uttered while seeing the subject together with the hearer). Wa readily goes with a formal verbal and it can be used in some inferior-superior situations.

To sum up, declarative final particies, $z o, z e, s a, y o$, and $w a$, among other things, express different degrees of speaker's assertion, roughly in descending order (the difference between $z e$ and $s a$ appears to be less crucial as far as the degree of assertion is concerned), and the use of the particles is 
contingent on the rank relationship between the speaker and the hearer: more assertive particles are more easily available to those with superiority.

\section{Confirmative and Dubitative}

In addition to the above declarative particles, there are three other particles common in standard Japanese: $n a, n e$, and $k a$. The first two, $n a$ and $n e$, emphasize the speaker's intention to involve the hearer in a truth-seeking speech act because the speaker may either be really uncertain about the statement or be afraid of appearing too self-righteous. Such particles are almost always pronounced with a rising intonation and are comparable in function to English tag-questions. When the speaker's insistence on the hearer's participation is extreme, $\mathrm{S}$-na/ne has almost the same illocutionary effect as that of interrogatives.

Applying the same ranking framework as we used for declaratives, we can determine that $n a$ is more dominant and assertive than $n e$ : $n a$ can be used only toward an inferior or equal, while ne is appropriate to certain superiors as well (e.g., son-father). The combination of formality and $n a$ is typically used by men of high social rank, middle-aged executives or university professors, for example, to their equals and tends to show prestige and informality simultaneously. When formality is added to the $\mathrm{S}$ in $\mathrm{S}-n e_{\text {, on the }}$ other hand, the formality overrides the friendly tone of ne to some extent and makes $S$-ne available in more inferior-superior situations. A young company employee would be able to say (a) to his superior, but not (b) or (c), in the following example, without provoking any emotional friction:

(3)-(a) Sutoraikiga kaiketsu-shi-mashi-ta ne. Strike SUBJ resolve do- FML PAST FP The strike is resolved, isn't it?

(b) Sutoraikiga kaiketsu-shi-mashi-ta na.

(c) Sutoraikiga kaiketst-shi- ta ne/na.

The last particle to be discussed is $k a$, which I prefer to call dubitative rather than interrogative, because it also occurs in certain declarative sentences pronounced with a falling intonation. ${ }^{9}$ Sentences with the form [S - ka ( $)$ ] are different from sentences with $n e / n a$ in that the latter presupposes belief in the statement rather than doubt while such a presupposition is lacking in question sentences with $k a$. Since $k a$ is unique as a dubitative, it cannot be compared with other particles with respect to assertion. However, 
question sentences can be formed without using $k a$ simply by pronouncing declarative sentences with a rising intonation. For example, sentence (1)-(a) could mean "Did Masao talk to Kazuko (about it)?" if the subject is marked as the topic ${ }^{16}$ and if the end of the sentence is pronounced with a rising intonation. Then, by comparing [S $-k a(f)]$ with $[S-f(y)]$, one can confirm that $k a$ has a relatively strong assertion. [S $-k a f \neq l$ ] (where [S] has no formal suffix) can be used only towards inferiors or equals while [S- $()$,$] is$ acceptable in certain inferior-superior situations such as son-father and junior student-senior student. Bach and Harnish (1979) point out that questions are a kind of direction: asking a question is to direct the hearer to answer. One can safely assume that dubitative $k a$ pronounced with a rising intonation is a grammatical marker for interrogative mood, the most direct indication of the illocutionary act of asking questions, but that rising intonation placed on the declarative sentence can at best suggest the speakers uncertainty, which may be interpreted by inference as a sign of interrogation.

\section{Degrees of Assertion}

The final particles that speakers of standard Japanese commonly use can be summarized for our purposes in the following table (particles near the top of the column are more assertive than those near the bottom):

$\begin{array}{ccc}\text { DECLARATIVE } & \text { CONFIRMATIVE } & \text { DUBITATIVE } \\ z o & n a & \mathrm{~kat} \\ z o / s a & & \\ w o & & \\ w a & n e & \end{array}$

It is certain that final particles involve various components of language performance, very few of which are known to us at the moment. But there is no doubt that the property we discuss here as "assertion" represents a linguistic correlative of power in society, a complex of a number of social variables. To assess the power of others and comply with the social rules based on the power relation is of particular importance in Japanese society, as Nakane (1970) emphasizes. The power relation between the speaker and the hearer governs the choice of a particle and the particle chosen in turn indicates the power relation. An utterance with an FP of strong assertion may make the hearer believe that the speaker is really superior. It is important to note that the rank relationship established between two individuals in a certain social situation applies to all circumstances involving the two, and 
it remains unchanged as long as the relationship between them exists. The superior-inferior relationship established between a university professor and his student would be extremely difficult to cancel or reverse even when the former student becomes the professor's colleague.

\section{Final Particles in Female Speech}

Turning now to the difference between male and female speakers, we note that the assertion measure established thus far seems to have quite a different relevance for women. Women have very limited access to $z o, z e, s a$, and $n a$. They can use only $y o, w a, n e$, and $k a$, either with formal predicates or other restricted modifications. Assuming that the assertion measure based on men's practice is basically correct and that there is only one system for all the speakers of Japanese regardless of sex, "we conclude that the discrepancy in the use of final particles between male and female speakers is in accordance with the social gap: women, being the inferior sex, use only those particles with weaker assertion.

The least assertive declarative particle $w a$ is in fact very rare in male speech, but it is the only one that women can use with little modification. Sentence (1)-(b), which 1 repeat here as (4)-(b) for the sake of comparison, is not quite appropriate for a woman to use, while (4)-(a) appears to be typical of female speech:

(4)-(a) Masao ga Kazake ni hanashtita wa.

(b) Masao ga Kaziko ni hanashi-ta ye.

Masao talked to Kazuko (about it).

Quite interestingly the gap in use for wa between male and female is not only in frequency but also in intonation. Women. especially younger women, usually pronounce wa with a rising intonation, while men always pronounce it with a falling intonation. Thus. Bokw ikt wa ( $/$ ) "I (male) will go" is not acceptable because of the conflict between the maleness on boknt, I (male), and the femaleness of wa ( $\$$ ). Kitigawa $(1977)$ has pointed to this difference between male $w a(\alpha)$ and female $w a(1)$ and attempted to defend Lakoffs claim that English-speaking women tend to use a rising intonation, suggesting that they are, or are supposed to be, uncertain about the content of their utterance, against the criticism by Dubois and Crouch (1975). He explains, "Femininity associated with sentence particle wa... derives from the speaker's attempt to reduce the strong sense of insistence inherent in this particle by expressing it with the gentle question intonation, thus submit- 
ting, in a manner of speaking, the appropriateness of that insistence to the better judgement of the addressee" (Kitigawa 1975:287). The fact that wa is in fact not as assertive as Kitigawa implies does not impair his argument but rather reinforces his position: even the least assertive declarative particle must be pronounced with a rising intonation in order for feminine tone to be definite.

Pronouncing $w a$ with a rising intonation is certainly characteristic of female speech, but the intention for using this marked intonation may not necessarily be to reduce the assertive force of wa. As mentioned earlier, wa is different from other declarative particles not only in the degree of assertion but also in the emphasis on the potential of the hearer's immediate response, which is essential to friendly conversation. In modern times, femininity is not solely a question of weak assertion but also a question of geniality. Modest but at the same time cordial speech will conform more perfectly to the idea of what is desirable in women than reserved and distant behavior. The use of rising intonation on wa may very well be a means of compensating for the weakness of $w a$ in this respect. However, uncertainty about the statement and intention to involve the hearer are two sides of the same coin, and whichever the case of wa may be does not really matter. Emphasizing her intention to involve the hearer in the truth-seeking procedure, whether from real uncertainty or from courtesy, makes the speaker sound more feminine.

In contrast to $w a, y o$ and ne express the speaker's intention to involve the hearer to such an extent that they can be used by women only in combination with wa. Combination particles wayo and wane are for women's use only. Thus, even at not so assertive levels of speech as those manifested by $z o, z e$, and $s a$, sentences are distinctively marked for the sex of the speaker. The following examples show the male and female contrasts at such levels:

(5)-(a) Masao ga Kaztko ni hanashi-ta wa. (2). Male

(b) Masao ga Kazuko ni hanashi-ta wa. (\$). Female

(6)-(a) Masao ga Kazuko ni hanashi-ta yo. Male

(b) Masao ga Kazuko ni hanashi-ta wa yo. Female

(7)-(a) Masao ga Kazuko ni hanashi-ta ne. Male

(b) Masao ga Kazıko ni hanashi-ta wa ne. Female

Dubitative $k a$ pronounced with a rising intonation is, as already mentioned, the most straightforward marker for questioning, i.e., demanding answers of the hearer. Question sentences like (8) are used only by male speakers with sufficient authority or between males with an especially close relationship. 
(8) Masao wa Kazako ni hanashi-ia ka( 7 )?

Questions that are more commonly used by males in informal situations are those marked only by rising intonation, i.e., the $[S-f(x)]$ pattern. This pattern may be used by some young women in very informal situations but it is not typical of women's speech. Most women use the pattern ending with nominalizer no pronounced with a rising intonation, which I shall discuss in the next section. That is, women can use [S $-k a(f)]$ question sentences only if the [S] is formal.

Males have access to the entire gamut of declarative particles and can express assertion in the most straightforward manner available in the language, but women barely express assertion and, if so, in the subtlest manner possible, by combining particles of weaker assertion or changing the intonation from falling to rising. In the following discussion on the final modes involving syntactic modifications, the tendency of female speakers toward less assertion will be further strikingly demonstrated. Syntactical rules that are to be obligatorily or optionally applied only by women all have the same effect of reducing the degree of assertion.

\section{Copula Deletion in Female Speech}

In Japanese there are three copula variants: $d a$, dest, and deari. Copula sentences ending with dearu reject the FP and naturally are very rare in conversation. The polite version desu is not formed by the regular process of adding a performative honorific suffix masu/mashi, but it is a suppletive form. In contrast to deari, da is colloquial though not formal at all, and it occurs in conversation with or without an FP. The following sentences (a) and (b), meaning "Masao is still a high school student" correspond in terms of speech level to the (a) and (b) sentences in (1):

(9)-(a) Masao wa mada kookoo- sei da. Masao TOP still high school student COP

(b) Masao wa mada kookoo- sei da yo.

Apparently, however, $d a$ by itself does not do what an FP does, namely, elicit the potential of the hearer's immediate participation. Sentences ending with $d a$ sound as brusque as (1)-(a), although they are used more frequently in conversation for reasons which will not be discussed here. ${ }^{12}$ As expected, copula sentences ending with da are exclusively for men. Women must add 
$w a$, preferably accompanied by a rising intonation. The final mode $d a$ wayo is acceptable in very informal situations, but $d a$ yo is improper in female speech.

\section{Fragments for Modesty?}

What is of particular interest here is the copula sentence pattern as exemplified in the following sentence, which is typical of female speech: $:^{1,3}$

(10) Masao wa mada kookto-sei wo.

This sentence, in which the copula is absent, is considered more proper for female speakers to say than the one ending with $d a$ wa or da wayo. Since it is the copula that carries the declarative mood of the sentence, deletion of the copula amounts to total loss of declarative mood. In fact, $d a$ is not a simple connector but has an extremely strong declarative sense, unlike regular verbs. As evidence for this, consider that while one can change sentences like (1)-(a) into questions either by simply pronouncing the final word with a rising intonation or by adding the dubitative $k a$ pronounced with a rising intonation, copular sentences like (9)-(a) do not comply with these transformations in either male or female speech: $d a$ can never occur in question sentences. It is this unusually strong declarative sense of da that makes sentences ending with $d a$ wa less desirable than the pattern [NP - NP $-y o$ in female speech in spite of the weakness of assertion of wa. The reason that it is not wa but yo that occurs after is because wa in general does not attach to elliptical sentences (perhaps for historical reasons), while $y o$ can occur with any fragmentary utterance or even intrasententially. ${ }^{14}$ The fact that the declarative force of $d a$ is felt as too strong to be used by women, even when followed by the weakest declarative FP pronounced with a rising intonation, seems to illustrate the submissive image that has long been imposed on Japanese women.

Application of the rule of copula deletion to common complex sentence patterns especially in conversation, yields a final mode that provides women speakers with the most feminine, submissive means of uttering declaratives:

(11)-(a) Masaoga Kaztko ni hanashi-ta no. Masao SUBJ Kazuko to talk- PAST NOM Masao talked to Kazuko.

(b) Masao na mada kookoo-sei na no. Masao TOP still high school student COP NOM Masao is still a high school student. 
The no in these sentences, which are used only by females. appears to be an FP, but it has been noted by most scholars of Japanese that the no in this pattern is related to the nominalizer no observed in sentences like:

(12)-(a) Masaoga Kazuko ni hanashi-ta no wa machigai da. Masao SUBJ Kazuko to talk- PAST NOM TOP mistake COP It is a mistake that Masao talked to Kazuko (about it).

(b) Masaoga mada kookoo-sei na no na akiraka-da. Masao SUBJ still high school student COP NOM TOP apparent It is apparent that Masao is still a high school student.

The na preceding the nominalizer no in (12)-(b) is a variant of copula da and is regularly used in this adnominal position. The same form is found in (11)-(b), indicating the nominal status of the following no. There is no doubt that sentences in (11) are nominal sentences. But why do women use nominal sentences? Martin (1975) suggests:

Women often use these nominalizations as full sentences-just as they often indulge in sentence fragments. . . left dangling for modesty or other reasons. (p. 945)

This leads us to a further question: Why does nominalization have the effect of giving a tone of "modesty"? I propose to analyze [S-no] as basically the same pattern illustrated by the following sentences:

$$
\begin{aligned}
& \text { (13)-(a) Masaoga Kazuko ni hanashi-ta no } \quad\left\{\begin{array}{l}
d k \\
d k w
\end{array}\right. \\
& \text { Masao SUBJ Kazuko to talk PAST NOM COP } \\
& \text { (b) Masaona madakookon-sei na no }\left\{\begin{array}{l}
\text { da } \\
\text { desut }
\end{array}\right. \\
& \text { Masao TOP still high school student COP NOM COP }
\end{aligned}
$$

Kuno (1973) calls these sentences "no dest construction" and compares them to English sentences of the form [It is that [S]]. This is an emphatic construction that puts part or all of the [S] in focus as new information. Thus, the [S] in the [[S]-no-da/desit] is typically a reason or an excuse for what has been said (old information). Given the rule of copula deletion we have discussed, [S-no] sentences in female speech will be most naturally accounted for by assuming that [S -no] is syntactically and semantically related to the emphatic construction [[S]-no-da/desu]. Although it looks like a fragment, lacking the matrix verb, such an accusation as represented 
by the following remark by Jespersen (1920) is not applicable, at least in this case:

I think that women much more often than men break off without finishing their sentences, because they start talking without having thought out what they are going to say. (p. 250)

By deleting the final copula from the $[[S]-n o-C O P U L A]$ construction, the speaker loses no content information. It seems that she not only knows perfectly well what she is going to say but has also considered the situation, assessed the interpersonal relationship, and applied additional rules to get her message across within the conventional framework of vertical society.

Such overprudent expression, however, may sound somewhat distant due to the absence of any sign of the speaker's intention to invite the hearer to participate in the conversational procedure. Addition of $y o$ to the [ $[\mathrm{S}]-n o]$ pattern can remedy this weakness and make utterances more cordial.

To recapitulate, women apply the rules of copula deletion because the aggressive tone of the informal copula $d a$ is da maging to the feminine image. By deleting it, women lose nothing but rather gain a social value of femininity.

\section{Directives in Female Speech}

As Austin (1962) pointed out, there are certain conditions necessary in order for an utterance to be felicitous, i.e., for the intended illocutionary act to be successfully performed. Such conditions will differ according to illocutionary types. To take a well-known example, one cannot christen a ship simply by uttering the sentence, "I name this ship "Queen Mary": the person who performs the act of christening must have the authority to do so and must adhere to the proper protocol. One of the necessary conditions for a directive utterance to be felicitous is that the person who performs the direetive act must have the authority to make the hearer act as directed. To issue a command or order-the strongest type of directive-one must have authoritative power over the hearer.

Granted that women are treated as the inferior sex in most modern societies, one would naturally expect that men and women would behave differently in directive acts. Key (1975) notes:

Females use alternatives to the imperative construction, which is the simple direct way of ordering an action: "Bring that here!" "Write that down!" "Have 
my suit cleaned!" Women use constructions which are not so straightforward. Questions substitute for commands: "Would you drop this by the cleaners on your way?" Modals soften the approach: "You could include that in the paragraph if you like."(p. 76)

Japanese provides a good example in support of this point: imperative constructions morphologically marked for imperative mood are not used by women unless they are also marked for formality In contrast, elliptical constructions in which imperative mood is absent are used either exclusively or preferably by women.

\section{Straight Imperatives}

There are a set of morphological rules which impart to the verb an imperative force: the imperative form is made by adding $e$ to the consonantending verb-stems and ro to vowel-ending verb-stems (the verb "to come" is an irregular verb so not subject to these rules). The following simplest sentences may serve as examples:
(14)-(a) Kutsuo hak=u. shoes DO wear (NON-PAST)
(I) wear the shoes.
(b) Kutsuo hak=e. shoes DO wear+IMP Wear the shoes.
(15)-(a) Kore o iabe-ru. This DO eat (NON-PAST) (I) eat this.
(b) Kore o tabe $=$ ro This DO eat + IMP Eat this.

(The verb form with $u / r u$ after the stem is traditionally called Shutshi-kei, finite form, and is typically used in the final position of nonimperative independent clauses and in some embedded clauses.)

The imperative construction with a thus-formed imperative verb has the most direct sense of command and is favored where precision is the predominant concern. Military orders to a troop of soldiers given by the commander-in-chief in marching drills are typically of this type; less stringent forms of this pattern of command are widely practiced in routine drills of gymnastics classes from elementary to high school and in many other social groups such as policemen's and firemen's groups. For this forceful pattern of command to be used at a more personal level, the speaker must not only be superior or at least equal to the hearer but also have an informal, intimate relationship with him/her. Women never have the privilege of giving directions in this straightforward manner. Even when speaking to her 
child, a woman does not use this construction. She must use polite imperatives like the following:

(16) Kutsuo haki-nasai.

Shoes DO wear-FML+IMP

Wear the shoes.

(-nasai is the imperative form of nasar $=u$, a subject honorific suffix, i.e., the suffix to express politeness towards the subject.)

This formal, but direct, imperative is used toward inferiors or equals but it does not require closeness or intimacy.

\section{Benefactive Imperatives}

In addition to these direct imperatives, Japanese has a peculiar imperative construction whereby the speaker can give directions in the manner of asking a favor. Compare (14)-(b) with the following "benefactive" imperative sentence:

(17) Kutsito hai-te-kur=e.

Shoes DO wear-\&-GIVE =IMP

Wear the shoes (for me).

This construction expresses imperative mood via the same morphological method as the one in (14)-(b). The hai-te-kure is a serial verb pattern [Verb-te-Verb], where $t e$ is a conjunctive particle conjoining the two verbs. The second verb kure, the imperative form of kureru, to give, can be used as an independent verb as well, but here it functions as an auxiliary to denote that the speaker is asking the hearer to do the directed action for the benefit of the speaker. This imperative has a much softer tone of authority, but its use is still restricted to a male who is superior or at least equal to his interlocutor. The formal version of the benefactive imperative that the following sentence exemplifies becomes more generally available to both men and women:

(18) Kutsuo hai-te-kudasai.

Shoes wear-\&-GIVE+FML+IMP

(Please) wear the shoes. 
The second verb in the [Verb-te-Verb] serial kudasai is a slightly irregular imperative form of kudasarti, a polite verb of giving. This pattern, being both formal and benefactive, can be used in some inferior-superior situations, although the relationship between the speaker and the hearer must be more or less formal: e.g., secretary-boss, student-professor, and wife-husband (the wife-husband relationship can be formal or informal in Japanese society).

The feminine equivalent of the informal benefactive imperative (17) will be the following:

\section{(19) Kutsuo hai-te-choodai. Shoes DO wear-\&-GIVING Wear the shoes (for me).}

The benefactive element choodai in this pattern has no grammatical indication of imperative mood. Strictly speaking, choodai is only the stem of a verb choodai-suru, to receive, consisting of a noun of Chinese origin and a Japanese verb sum, to do. ${ }^{15}$ For some reason, which is not our present concern, this stem of a verb meaning "to receive" came to be used both in the [Verb-te-Verb] serial context and in the main verb position as if it were the imperative form of a verb of giving. ${ }^{16}$ This elliptical and aberrant benefactive imperative is used exclusively by women in informal situations, but it is not a simple female counterpart of (17). While the male benefactive imperative is used only towards an inferior or equal, imperatives like (19) can be used in inferior-superior situations if the inferior has an intimate relationship with the superior. When it is used in an inferior-superior situation, however, it is pronounced with a rising intonation and it takes on a coquettish, importuning tone. The pattern will be very effectively used when, for instance, a young girl asks her father to buy her a new dress.

\section{Female Imperative}

There is another pattern which may be taken as a variant of the benefactive imperative although the benefactive element which would carry imperative mood is completely missing, leaving $t e$, the clue to it, behind. Compare the following with the above benefactive imperatives:

(20) Kutsuo hai-ie. shoes DO wear-\& Wear the shoes. 
When this pattern is accompanied by an obvious falling intonation, it has a fairly strong directive force and can be used by men as well as women in superior-inferior situations. In most contexts, however, women pronounce this imperative pattern with a rising intonation or they add $y$ to it, which men are not supposed to do. The motivation for these modifications should be clear by now: the rising intonation expresses uncertainty and $y$ geniality.

One can perform directive acts with constructions other than imperatives. Such constructions all seem to exhibit the same pattern of sex differentiation. Interrogatives in the form of [S -ka(, 1$)]$, having an overt interrogative marker, have a more straightforward force of direction than the [S - ( ( ) ] questions. It has already been noted that the former pattern is not used by women unless formality is expressed on the [S]. Hortatives without FP or those with ze, yo, na are all masculine, i.e., exclusively for men's use. The only hortative pattern that women may share with men is the one with confirmative FP ne besides formal hortatives.

The explanations for all these sex-oriented restrictions on the use of imperatives, interrogatives, and hortatives must be essentially the same. Women are generally ranked as inferior in Japanese culture, in which ranking is a crucial determinant of interpersonal behavior, and so it is in linguistic behavior. The exclusiveness of many sex-oriented rules in the language is a reflection of the inflexibility of the ranking phenomenon in the society.

\section{Politeness and Female Speech}

It has been pointed out in several articles on sex differentiation in language that politeness is characteristic of female speech: women are supposed to talk more politely than men. But what is politeness? How can it be discussed meaningfully in linguistic terms? We need a good working definition of politeness to deal with the question of its relation to sex differentiation. In the following analysis of Japanese honorific usage in female speech, I shall refer to Lakoff's (1975) "rules of politeness," which holds that there are three rules of politeness (formality, deference, and camaraderie) which interact with the "Rules of Conversation" suggested by Grice (1975). The rules of politeness appear to be quite relevant for our analyses due to the existence of grammatical categories in Japanese that neatly correspond to those distinctions. The politeness expressed by performative honorifics (formal forms) corresponds to the first two, formality and deference, and the conversational intention expressed by final particles to the rules of camaraderie. First, let us 
distinguish performative honorifics from propositional honorifics (subject and object honorifics) according to Harada (1976).

Performative honorifics are those which reveal the speaker's formal attitude towards the hearer by use of performative honorific verbs (most honorific verbs are formed by adding the performative honorific suffix masti/mashi to the main verb, but there are more than several verbs which have suppletive honorific forms). Subject or object honorifies are those which indicate, via verb morphology, that the subject or object is a person who is socially superior to the speaker (SSS).

Theoretically, all of the three honorifics can exist within the same sentence but combination of the two propositional honorifics are difficult in practice and occur very rarely if at all. Performative honorifics can smoothly combine with subject or object honorifics. The following examples should be sufficient for the present purposes. (Cf. Harada for more detailed and formal analysis of these honorifics.)

(21)-(a) Shachoo (SSS) ga Tanaka-sanni o-hanashi-ni-nat-ta company president SUBJ Mr. Tanaka to talk (SUBJ HONOR)-PAST The company president taked to Mr. Tanaka (about it).

(b) Tanaka-sanga shachoo (SSS) ni y-hanashi-shi- ta. Mr. Tanaka SUBJ company president to talk (OBJ HONO)-PAST Mr. Tanaka talked to the company president (about it).

(c) Masoga Kazukoni hanashi-mashi- ta Maso SUBJ Kazuko to talk (PERF HONOR)- PAST Masao talked to Kazuko (about it).

(d) Shachoo (SSS) ga Tanaka-san ni o-hanashi-ni-nari-mashi-ta. company president SUBJ Mr. Tanaka to talk (SUBJ HONOR-PERF HONOR)-PAST

The company president talked to Mr. Tanaka (about it).

(e) Tanaka sanga Shachoo(SSS) nio-hanashi-shi-mashi-ta.

Mr. Tanaka SUBJ company president to talk (OBJ HONOR-PERF HONOR)-PAST

Mr. Tanaka talked to the company president (about it).

Since performative honorification is the type which is particularly relevant for concern with interpersonal relationships, with which we are most interested at the present moment, we shall use the term "formality" or "formal form" only in reference to performative honorifics, and use more specific 
terms (subject and object honorifics) when we mention other types of honorification. ${ }^{17}$

Formality in Japanese is not something that individuals can use as they wish, but it must be applied according to the same set of social rules of interpersonal behavior that we have been referring to in the discussion on final particles as well as other elements that comprise the final mode. With the addition of formality the picture of the final mode becomes more or less complete and naturally more complicated, and the complication requires us to give a more careful look at the phenomenon of social ranking. It seems to be helpful to discern two types of criteria used for identifying the ranking relationship between individuals participating in interactions, drawing on the concepts that Nakane (1970) proposes-attribute and frame: "Attribute may be acquired not only by birth but by achievement" (p. 2). An individual who was born in a family of a high social class has attributes characteristic of the class. An executive member of a large corporation has attributes of the group of executives even if he is from a poor working-class family. "Frame may be a locality, an institution or a particular relationship which binds a set of individuals into one group; in all cases it indicates a criterion which sees a boundary and gives a common basis to a set of individuals who are located or involved in it" (p. 1). For the purpose of our discussion of linguistic interaction, we may use the term "frame" in a slightly extended sense. For example, an executive of a big corporation will be ranked as superior according to his attribute and he may be given prestige by society, while a worker of his company with no supervisorial capacity will on the other hand be considered inferior. We may distinguish arbitrarily three social strata-high, intermediate, and low-which are crucial for the attribute criterion.

In conversational situations, the speaker's rank on the basis of the frame superimposes over the rank based on the attribute. An executive of high rank, for example, may be ranked as inferior in relation to a major stockholder with significant influence over the operations of the corporation, and an office employee with no title will be ranked superior when he talks to a janitor. The social significance of politeness is derived from both ranking criteria.

If the speakers are both of the highest rank, they may use formal forms. which are often coupled with the most assertive FP zo/na, indicating that they are formal/deferential but dominant. The combination of formality with intent to dominate a situation creates a somewhat dignified style (e.g., 13-(b))-(male) VIP register. If there is any noticeable gap between the two high-ranking personnel, the relatively superior one has an option between 
formal and informal speech, and if he chooses the latter the formality in his inferior colleague's speech signifies deference. ${ }^{\text {Is }}$

Where there is an equal relationship between speakers of intermediate rank, formal verbals followed only by those particles with milder assertion, $y o, w a$, and $n e$, may be reciprocally used. If one of the speakers is slightly superior, only the inferior will use formal speech. In this case, too, the formality of the inferior's speech may be taken as a sign of deference.

At the two higher levels then, each rank is distinctly marked by virtue of the fact that at least one of the speakers uses stronger or weaker FP after formal sentences, respectively, and within each level, vertical relationships, if existing, may be differentiated from equal relationships by the absence of formality only in the superior's speech.

Between the two acquaintances of the lower rank, formality is normally unimportant. Both speakers will speak without expressing formality but with FP of milder assertion, namely camaraderie markers. Within this level, ranking based on the frame is less significant than in the other two classes. Showing that they are friends to each other will be sufficient. But if there is a need (seniority could be a reason), the superior may lift his status up by using more assertive particles than the inferior. When the two speakers of the high or intermediate rank are not only equal but personally very close to each other (e.g., colleagues of the same age and from the same college), the same camaraderie pattern dominates: both might speak without politeness but with strong assertion. Nonpolite sentences with assertive $\mathrm{FP}, z o, s a, z e$, and $n a$, give a strong sense of camaraderie to the conversation when reciprocated. ${ }^{19}$

If the disparity between the two speakers is obviously great, the inferior had better speak with formality but without FP. An inferior's friendly approach to a superior may be construed by the superior as impertinence.

One could offer numerous counterexamples to this analysis of the relationship between ranking and politeness, but most will either tend to be more complex cases involving other variables than considered here (e.g., personality or diachronic factors) or anomalous cases. For example, an old janitor who has worked for the same company for forty years may consider himself an important witness of the history of the company and speak accordingly: he may use nonpolite forms to a member of the intermediate level, not to speak of a man of his own rank. But there will be social punishments for his anomalous behavior. His coworkers may not like him; new employees of the intermediate levels who do not know about his personal history may feel hostile toward him, and so forth.

Interesting at this point is the fact we noted earlier that in a vertical 
relationship, the rules of politeness must be abided by quite strictly by inferiors, but they can be flexibly used by superiors. Nakane notes:

Some flaunt their higher status by haughtiness towards inferiors and excessive modesty towards superiors; others may prefer to conceal haughtiness, remaining modest even towards inferiors, a manner which is appreciated by the latter and may result in greater benefit to the superior. (p. 31)

In other words, formality shown by a superior toward his inferior is interpreted as a virtue of the superior and helps the speaker gain the inferior's genuine respect while an inferior's politeness toward his superior is taken for granted.

\section{Women's Place}

Men are bound both upward and downward by vertical relationships. To know one's own rank and the rank of others is often more important than competency in work. Our question is: How are women's positions related to the male rankings? What does politeness mean to women in Japanese society? Attempts to apply this oversimplified sketch of ranking for women's speech may be problematic. When we start examining women's speech more closely, all the generalizations made based on men's behavior somehow do not hold. Women cannot use $z o$ and $n a$ and thus cannot speak as pompously as male executives. When a woman drops formality from her speech, she must also hold back assertion; i.e., female informal speech does not function as an index of relative superiority. However, a man of low rank will always speak politely to the wife of an intermediate or high-rank member. Therefore, one cannot characterize women's speech simply as a function of low social rank. It is true that they are never placed in the high rank but they do not clearly fit in the low rank either. It appears that women have no place in the society defined in terms of vertical relationship.

In constructing the theory of vertical versus horizontal society, to which I have often referred, Nakane did not fully explore the question of women's status in Japanese society and made a somewhat puzzling comment on Japanese women:

It is well known that Japanese women are nearly always ranked as inferiors; this is not because their sex is considered inferior, but because women seldom hold higher social status. Difference of sex will never be so pronounced in Japanese thinking as in America, where classification (though not for purposes of estab- 
lishing rank) is primarily by sex. I am convinced that in American society sexconsciousness predominates over status-consciousness. the exact opposite of lapan. (p. 31)

If Japanese people are not as sex-conscious as Nakane thinks. it is hardly because women are not treated as inferior in their culture, but rather because the sex differentiation has gone unquestioned for so long that it has been taken for granted. If more women become interested in doing the things that are presently monopolized by men and if they come to want to "hold higher social status," both men and women will not be able to avoid becoming sexconscious. Men will feel challenged and attempt to convince women that they are physiologically different from men and not capable of doing those things. And women would have to argue against them, which is certainly the situation in the "sex-conscious" society of the U.S., as it is in the emerging situation in Japanese society.

A woman's place in Japan is still "in the home." If she steps beyond the domestic boundary, she can find no acceptable place or rank to be identified with. Social rules intended for male members do not tell women how they should behave or speak in order to fit in to the establishment. Without identity outside the home, women have no choice but to talk formally and unassertively, as men would when they talk to strangers. When a woman talks to a man without using formal forms, her speech can mislead the man, the suggestion being that she is trying to establish an "intimate" relationship with him, although her intention is to talk to him as an equal, a friend.

As I have mentioned at various points in the foregoing discussion, formality brings female speech closer to male speech. Constructions of the type illustrated by (1)-(b), which can be used by women at the cost of criticisms or stares, become perfectly acceptable when the performative honorific suffix is added to the verb. Predicate nominal sentences with formal copula desu are available to women as well as to men. Formal versions of direct imperative and benefactive imperatives can be used by both men and women. With the exception of extremely assertive FP, $z o, z \mathcal{C}$, and $n a$, almost all the constructions that men use can be shared by females if formality is added. This does not mean, however, that women can talk on a par with men in real social situations by using formality in speech. In conversation between a man and a woman, if the woman talks in formal speech (assertive/ polite) and if the man responds in informal male speech (assertive/nonpolite), which is quite likely, the relationship between the two will take on a social significance reinforcing the idea that the woman is inferior to the man. If both stayed on the formal level, they will remain distant. There is no way a 
woman can talk assertively maintaining a spirit of camaraderie and express a relationship on the same footing with a man.

\section{Woman-to-Woman Talk}

What happens in conversation between two women is not the same as we observed between males. Women who meet for the first time will talk politely to each other, as men do in the same situation. But very quickly they will switch their level of speech from formal to informal and talk as if they have known each other for a long time, instead of changing the equal-distant situation to a vertical camaraderie relationship, which men do in most contexts. The following comment made by a male university professor at a round-table discussion on women's language is quite indicative.

I envy women. In the case of women, even in a situation where they are meeting for the first time, they talk formally only at the very beginning and they quickly switch the level to an informal one, saying, for example, "... na no yo." This shows that women talk to each other informally, regardless of age and seniority. ${ }^{20}$

The same speaker goes on to point out that vertical relationships established on the basis of seniority and other variables between men remain unchanged for years. During this time, men have to use proper levels of speech in every circumstance. It is interesting to note that men sometimes feel straightlaced within the social structure and look at women's casual nonassertive talk with envy. Gender constraint is harmful to men as well as women, although men, being the dominant group, may not be distressed by it to the same extent as women are.

Women are considered inferior to men, but women do not consider themselves to be all equal to each other. They are prone to believe that the rank of the men they are tied to, fathers and husbands, is their own, and if the rank of those men is high, they try to distinguish themselves from other women by using excessively formal language. For example, compare the following with (7):

\section{(22)-(a) Masaoga Kazukoni hanashi-mashi-tano.}

Masao SUBJ Kazuko to talk-POLITE-PAST NOM

(It is) that Masao talked to Kazuko (about it).

(b) Masaowa mada kookoo- sei desut no. Masao TOP still high school student COP (POLI IE) NOM (It is) that Masao is still a high school student. 
Formally speaking, the clauses followed by no are embedded clauses, which do not freely allow a performative element. This restriction on nominal clauses is a phenomenon widely observed in world languages and makes sense. But it seems that such linguistic logic does not matter to women speakers. They ignore the constraint and use the performative honorifics in this nominal construction anyway so that their speech will manifest the prestige of high-class background. This tendency may be carried to almost absurd extremes in expressions like the following:

(23) Masaoga Kazuko ni hanashi-mashi-ta-n-zaamasu no. Masao SUBJ Kazuko to talk-FML-PAST-NOM-COP (SUPER POLITE) NOM

(It is) that Masao talked to Kazuko (abont it).

The zaamasu in this sentence is a contracted form of the superpolite copula (de)gozaimasu and is used only by relatively older women of high class. (The term "zaamasu lady" was coined to refer to those housewives of the upper class who constantly use this ending in their speech.)

Another example of female speech indicating background/shadow prestige is related to imperative expressions ending with conjunctive particle te.

(24) Kazuko ni hanashi-te.

Kazuko to talk-\&

Talk to Kazuko (about it).

This pattern is potentially quite ambiguous. When pronounced with rising intonation, it can be a question, meaning "Have you talked to Kazuko (about it)?" as well as an imperative. ${ }^{21}$ And if it is followed by yo, it can mean either "Talk to Kazuko (about it)," or "(I) have talked to Kazuko (about it)." This truncated pattern used in the nonimperative sense is considered to be extremely feminine and is used only by those women from "nice families." More typically, sentences of this pattern are accompanied by subject or object honorfics as shown below: ${ }^{22}$

(25) Kazuko ni o-hanashi-ni-nat-te ( $\boldsymbol{f}$ ). (Subject Honorific)

Talk to Kazuko (about it).

Have you talked to Kazuko (about it)?

(26) Kazuko ni hanashi-mashi-te yo. (Performative Honorific)

I have talked to Kazuko (about it). 
(27) O-too-sama ni o-hanashi-shi-te ( $\boldsymbol{t})$. (Objective Honorific)

Talk to your father.

Have you talked to your father?

(28) Kazuko ni o-hanashi-ni-nari-mashi-te ( $\rightarrow$ ). (Subject and Performative Honorific)

Have you talked to Kazuko (about it)?

(29) Kazuko ni o-hanashi-ni-nat-te-kudasai-mashi-te (x). (Subject Benefactive \& Performative Honorific)

Have you talked to Kazuko (about it)?

None of these expressions will be used by men except those who do not identify themselves with the conventional male image-gay men who consciously speak women's language regardless of class or age. It is only women who develop such elaborate and redundantly polite constructions. However, the speaker in these cases does not necessarily mean to be formal to the hearer in using these forms. Inconsistency with regard to formality in sentence (25) proves that the speaker is contradictory as to whether she wants to be formal to the hearer or not. The sentence is a subject honorific sentence, which is supposed to express the speaker's respect to the subject of the sentence (i.e., the hearer in the imperative), but it does not have the performative honorific suffix to show formality toward the hearer (i.e., the subject). This pattern is in fact heard quite often in very casual situations.

The final example that 1 shall discuss in connection with women's shadow prestige is cited in Harada (1976). In Japanese, nouns are often accompanied by an honorific prefix $O$ - to indicate that the object referred to by the noun belongs to a person who is socially superior to the speaker (SSS). However, Harada comments, "There are nouns that have honorific shapes but whose appearance is not conditioned by an occurrence of an SSS in the subject, object, or possessive." To cite one of his examples:

(30) O-biinu ikaga?

HON-beer how about

How about some beer?/ Would you care for some beer?

Hadada explains "beautificative honorifics" like the above as follows:

Beautificative honorifics have a nontrivial stylistic effect of making the utterance sound soft and feminine. Thus, their absence is usually taken as a token of virile speech. Their abuse is a favorite means of caricaturing a snobbish 
middle-class housewife who tries to boast of her education and good manners. (p. 542)

It is clear that the purpose of using honorifics in women's speech is quite often to indicate one's husband's or father's high rank rather than one's own. Women are as rank-conscious as men are in Japanese culture, but since they have no ranks themselves, they tend to compensate by manifesting their family rank by exaggerated honorific usage.

In summary, the hierarchy obtaining among Japanese males and the rules based upon it do not apply to women as "nonpublic" individuals. Therefore, formality which is used to indicate rank relationships among men, tends to have different significance among women, where it is used to indicate the rank of the men they belong to as much as to express genuine formality or deference. For men, formality is an indicator of their own rank or genuine deference.

\section{Future of Female Speech}

Onna Daigaku (College of Women), quoted at the beginning of this article, served as a guide for Japanese women for two hundred years during the feudal age (seventeenth to nineteenth centuries) and has persisted well into the modern age. It has not been more than two or three generations since women were taught to follow literally the lessons of Onna Daigaku in girls' schools. Like many other countries, however, Japan has undergone unprecedented and widespread change during the postwar period and so has the consciousness of women. Sanae Kubota, a socialist member of the House of Councilors, recalls, "When I was in a girls high school, I read Shin Onna Daigaku by Yukichi Fukuzawa, which contained Onna Daigaku by Ekiken Kaibara. Upon reading it, I became so furious that I threw the book against the wall." 23 Four-year universities under the new higher education system put into practice after the war graduated over a million women students by 1980 , the first woman ambassador was nominated in 1980, and there are more than several women who have been active in politics or business. The number of women principals of elementary schools has greatly increased during the past 15 years. However, we have seen that sex-differentiation in Japanese is quite exclusive, and female speech does not accommodate a female superior with a means by which she can talk to her subordinates in such a superior manner as a male superior would do. A woman in a high position, therefore, is thrown on the horns of a dilemma, between the needs of authority demanded by her status and her feminine identity: if a woman 
leader talks in female style without formality to her subject, man or woman, she may fail to be assertive enough, and on the other hand if she talks formally, she may sound too distant. The spirit of camaraderie is a key to success as a group leader in Japanese society. The personal tie between the leader and the subjects is essential for the integrity of the group and "strong, functional personal ties always derive from the informal structure" (Nakane 1970:64). How those already successful women are handling this contradiction is an extremely interesting and relevant question for linguists as well as sociologists. There will not be a uniform solution to this problem immediately applicable for all women in higher positions. The problem will have to be coped with individually, depending on the situation and the personality.

The problem we are facing here is delicate and perhaps has not yet been clearly recognized as such by women themselves. Novelists have not started to write about such women, and movies are still structured on the basis of traditional concepts of human relationships. The only example of dialogue between a female superior and a male inferior that has come to my attention is from a short article written by Ryooko Ozawa in 1973. Ozawa was elected unanimously as a city council member of a large satellite city of Tokyo in 1971 and has been pursuing her political career with vigor. She writes that female speech is a product of the idle life of housewives and does not serve those who must work in situations where there is tension. In her own position, where quick and proper decisions are urgent, she notes that her own speech tends to become masculine as she tries to convey her ideas as precisely and emphatically as possible. Her point is proven by a dialogue that took place between Ozawa and her male secretary, although there seems to still be a limit to the degree of masculinization. The traditional male and female speech patterns appear almost completely reversed in the example: Ozawa blatantly uses final particles yo and ne without the normal modifications required by standard female speech. She even uses the masculine confirmative particle $n a$ (though perhaps not in the standard confirmative sense but in an exclamatory sense-as if talking to oneself). Being well aware of the nature of female speech, Ozawa chooses to give priority to her role as a council member over her feminine role, at least in her office. The approach exemplified by Ozawa, however, may not be acceptable to other women. The association between male speech and manhood or between female speech and womanhood has been so strong in Japanese culture that use of male speech by a woman tends to be taken as an intention to claim manhood rather than as an intention to fulfill her social role as well as men. In order for this approach to be more generally applicable, there must be a change in 
the total image of masculinity and femininity and in the interpretation of the existing stylistic variations, so that either men or women can use any style according to the role that they are playing in different social situations. As a matter of fact, the way younger Japanese talk today suggests that the change is already under way. There seem to be very few young men, at least among college students, who use $z o$ and ze with serious intention to assert themselves, although they may use these particles with a joking or humorous tone. They still use sa and na quite frequently but not as often as ne, the weaker and friendlier confirmative. Imperative sentences like Kazuko ni hanashi-te yo, Talk to Kazuko (about it), which used to be taken as a "sissy" expression, is used quite naturally in many contexts. Similarly, interrogative sentences of the pattern [S -no ( $\Rightarrow$ )] are even preferred to those with the pattern [S-ka ( )]. The [S -no $y o$ ] or [S -wa yo] pattern is still limited to women but [S no ne] is common. ${ }^{24}$ That is, the patterns which are in the borderline area of male/female distinction are gaining popularity among young male speakers. The opposite tendency is observed in the way younger women converse. Sentences of the [S - yo] pattern are used with less hesitancy than before. Sentences with no FP (e.g., (1)-(a)) occur frequently in certain discourse contexts. Sentences ending with the masculine copula $d a$ and FP yo are particularly common. Informal hortatives with yo or dubitative $k a$ have almost lost their masculine connotation in informal speech. A man who speaks at an extremely masculine level would seem almost boorish on many occasions. Similarly, extreme femininity in a woman's speech would be interpreted as a sign of either a lack of modern education or certain professions traditionally associated with women (e.g., bar hostess). It is a remarkable fad among teen-age girls in Tokyo and some big cities to use boku, "l", which has always been used only by men, instead of atashi, the female first person pronoun. The reason is, the girls explain in a TV program discussion, that they cannot play an even game with boys if they use atashi. Jugaku (1979), who participated in the program as a commentator, points out that it is not only the word " $I$ " but also the final mode and pronunciation that are consciously masculinized in their language. They no longer say ". . no yo" or ". . . wa yo." It is evident that the general tendency is increasingly toward overlapping of male and female speech. This change in language must be ascribed to the changes in the image that younger people have of masculinity or femininity. Men and women are no longer separated as strictly as they used to be and they even enjoy each other's company in areas beyond marital or sexual relations.

"Every language is integrated with the culture in which it operates, and its lexical structure (as well as at least part of its grammatical structure) 
reflects those distinctions which are (or have been) important in the culture" (Lyons 1977:248). I have attempted to show (1) that Japanese distinguishes several levels of speech reflecting various rank relations held between the speaker and the hearer; (2) that the levels that female speakers have recourse to are not able to simultaneously express assertion and geniality; and (3) that, as a result, women are linguistically hindered from being as successful in higher positions as men. I have suggested that expression will assume different social values as the concept of male-female relations changes from the type represented in Onna Daigaku to the type prevalent among the younger generations. Some of those who are now among the young may turn back to the traditional order as they grow older but some others will remain as they are. The tendency toward equality will then eventually permeate the entire culture, and new stylistic variations will be created to accommodate newly emerging values.

\section{Notes}

1. Onna Daigaku (College of Women, or literally Important Great Lessons for Women) is a moral textbook compiled during the era of Edo. Originally the author was believed to have been Ekiken Kaibara (1630-17/4), a Confucian. But his authorship has recently been questioned by Japanese scholars. Encyclopedia Japonica (Shogakukan, 1968) conjectures that one of the chapters of Ekiken Jikkun (Ekiken's Ten Lessons) entitled Joshi o Oshiant no Hoo (The Method of Educating Women, which is almost the same as Orna Datgaka in content, was used as a text for women's education and later it was made an independent book and was given this title.

2. Formality and politeness are not always clearly distinguished. In this study $I$ use formality to refer to the value of formal forms of verbals as opposed to informal ones which are explicitly differentiated in Japanese. By politeness 1 mean a sharing, unintruding, tinaggressive atitude which is mostly expressed with final particles or by intonations in Japanese. Alse see Lakoffs discussion of this distinction, which is briefly introduced in this study.

3. In one-sided speech situations like lectures, therefore, final particles are very rare although formality is preferred.

4. Among the modern novels that I have surveyed, those by Keita Genji were particularly helpful. They are full of dialogues between various characters with various ranks in comapny situations. The examples cited in this study are from Tenka Taihet, by Genji.

5. Ze may have been derived from $z o$, which existed in the language throughout its history as an emphatic particle, plus $i$, a confirmative particle, which is no longer in use.

6. Alfonso (1976) explains, "This particle is used to indicate that something is OBVIOUS: it is equivalent to "naturally" (p. 1146).

7. Brown (1960) discussed the fact that the power semantic that prevailed in the nineteenth century is gradually giving way to the solidarity semantic and states that in dyads such as customer/waiter, officer/soldier, employer/employee, parent/son, and elder brother/younger brother, the probability of a mutual T-form (such as French speech using $t u$ instead of vous) has increased. As I discuss in the final section of this paper, Japan is also undergoing a change comparable to this, and the fact that we hear informal speech with $w$ reciprocated between father and son for example today may very well be a manifestation of the change.

8. The development of wa is not fully understood. If it is a residue of an exclamatory 
sentence final particle that has existed in the language throughout history, the fact that wa does not have the force of involving the hearer or reaching out to the hearer makes good sense: it indicates that the sentence to which it is attached is a statement made to the speaker himself/herself.

9. When $k a$ is pronounced with a falling intonation, S- $k a$ is not taken as a question. It expresses the speaker's surprise as in (a) and/or acknowledgement of [S] with uncertainty or hesitancy as in (b):

(a) Oh, ki- ta ka( $)$.

Ah, come- PAST FP

Ah, (you) have come!

(b) Sorejaa, yaruka ( $)$

then do FP

Then, (we) will do it, won't we?

10. Interrogatives are presuppositionally more complex than declaratives and they put some restraints on topicalization.

11. Hideo Suzuki (1976) proposes an analysis with two separate systems, one for the use of males and the other for the use of females.

12. The construction $[[\mathrm{S}]$ no $\mathrm{COP}]$ is very much preferred to $[\mathrm{S}]$ in conversation even where there is no obvious reason. The [[S] no COP] pattern does not require the FP so much as the [S] pattern, either. That is, many conversational sentences end with a copula da or desu, and of course it is men who use $d a$ in casual situations.

13. The pattern occurs in male speech also, but only very rarely and with a special connotation. Isago Mio (1958) says that it is pronounced with a different intonation by males and it sounds vulgar; the average male speaker would not use it.

14. Yo is similar to confirmative ne and na in this regard. For example, utterances like the following are common in formal conversation;

$$
\text { Kinoo }\left\{\begin{array}{l}
y o \\
n a \\
n e
\end{array}\right\} \text { Masaoga }\left\{\begin{array}{l}
y o \\
n a \\
n e
\end{array}\right\} \text { Yoshikoni }\left\{\begin{array}{l}
y o \\
n a \\
n e
\end{array}\right\} \text { hanashi-te }\left\{\begin{array}{l}
y o \\
n a \\
n e
\end{array}\right\} \ldots
$$

Yesterday, you know, Nasao, you know, talked to Yoshiko, you know, and . . Na and $y$ are used only by males in this context. Excessive use of these particles for hedging may be an indication of the speaker's extreme insecurity/anxiety.

15. There are numerous verbs of this pattern. Some of these verbs are:

$\begin{array}{ll}\text { benkyoo-suru } & \text { to study } \\ \text { ryoori-suru } & \text { to cook } \\ \text { sinyoo-suru } & \text { to trust } \\ \text { ryokoo-suru } & \text { to travel } \\ \text { kekkon-suru } & \text { to marry } \\ \text { shimpai-suru } & \text { to worry }\end{array}$

16. Choodai, Give me, may be considered to be an elliptical expression of Choodai shimasu (receiving do +FML), or causative constructions like Choodai-sa-se-te-itadaki-masu (receiving-do-CAUSE-\&-RECEIVE +FML) and Choodai-sa-se-te-kudasai (receiving-do-CAUSE-\&GIVE+FML+IMP).

17. In addition to the traditional pattern of subject honorifics discussed in Harada, a historically new pattern with the passive suffix rare is used quite commonly, perhaps even more often than the traditional subject honorifics, and it seems that male speakers have more oppor- 
tunities for using them. In Genjis novels I found only a few examples of rire-honorifics used by female speakers. If it is so, it is another piece of evidence that women talk more politely than men.

18. For example, in a conversation between the president and one of the executives of the same company the former probably speaks with informal sentences with or without $\mathrm{FP}$, while the latter speaks with formal sentences with assertive particles, possibly nit.

19. The main character of Tenka Taihe $t$ and his friend, who have developed a very special camaraderie relationship during the time when they were detained together in Siberia after World War JI, talk to each other at this level.

20. Takao Sofue. "Onna no Kthtoba: Otoko no Kotoba" (Female language: Male language), Gengo Seikatsu, No. 262, 1973.

21. There may be a slight difference in intonation between the imperative and inter rogative use of this pattern. I have asked several female speakers about the intonation of this pattern, but the responses did not clearly prove the difference.

22. These expressions are not easily relatable to complete sentences with the second verbal element after $i e$. It may be the case that conjunctive $t e$ in [Verb- $t$-Verb] was reanalyzed as a female speaker marker as a result of frequent use of the elliptical Verb-te.

23. Ai-Fonim (Women-Made Information Journal), no. 14 (Tokyo: ASA Kikaku), p. 30.

24. The following sentence is one of those uttered by a male student who was talking to other male students in my presence:

Boko anokito nikit-tanone. Demohakkiri kotac-te-kure-nakat-ta-w. I(male) that person to ask-PAST NOM FP but clearly answer \&-GIVE-not-PAST-FP I asked her, but she did not answer (for me) clearty.

\section{Bibliography}

Ai-Fortm (Women-Made Information Journal on Women's Issues), no. 14, Tokyo: Asa Kikaku. Alfonso, Anthony Japanese Language Patterns, vols. 1 \& 2. Tokyo: Sophia University L.L.. Center of Applied Linguistics, 1974.

Austin, John L. How To Do Things With Words. Cambridge, Mass: Harvard Iniversity Press. 1962.

Bach, Kent, and Harnish, Robert M. Linguistic Communication and Speech Acts. Cambridge. Mass.: M.I.T. Press, 1979.

Brown, Roger, and Gilman, Albert. "The Pronouns of Power and Solidarity" in Style in Langutage, ed. Thomas A. Sebeok. Cambridge, Mass.: M.I.T. Press, 1960.

Dubois, Betty L., and Crouch, Isabel. "The Question of Tag Questions in Women's Speech: They don't really use more of them, do they?" Language in Society 4, no. 3 (1975): 289-94.

Gengo Seikatsu, no. 262 (July 1975). Tokyo: Chikuma Shoboo.

Grice, Paul H. "Logic and Conversation," Syntar and Semanties 3. Spetch Att, eds. P. Cole and J.L. Morgan. New York: Academic Press, 1975.

Harada, Sinichi. "Honorifics," Syntax and Semantics S: Japanese Generative Grammar, ed. Masayoshi Shibitani. New York: Academic Press, 1976.

Jespersen, Otto. Language: Its Nature, Development and Origins, Chapter XIII, "The Woman," pp. 237-54. London: Allen \& Unwin, 1922.

Jugaku, Akiko. Nihongo to onna (Japanese and Woman, Tokyo: Iwanami, 1979.

Key, Mary Ritchie. MalefFemale Langiage. Metuchen, N.J.: Scarecrow Press. 1975.

Kitigawa, Chisato. "A Source of Femininity in Japanese: In Defense of Robin Lakoffs Langituge and Womans Place," Papers in Linguistics no. 10:275-98.

Kuno, Susumu. The Strtcture of the Japanese Langtage. Cambridge, Mass.: M.I.T. Press, 1975.

Lakoff, Robin. Language and Woman's Place. New York: Harper \& Row, 1975.

Lyons, John. Semantics, vols. 1 \& 2. Cambridge: Cambridge University Press, 1977. 
Martin, Samuel E. A Reference Grammar of Japanese. New Haven: Yale University Press, 1975.

Mio. Isago. Hanashi-Koloha no Bunpoo (Grammar of Spoken Language). Tokyo: Hoosei Daigaku Shuppan Kyoku、 1958.

Nakane, Chie. Japanese' Socict!: Berkeley: University of California Press, 1970.

Orasanu. Judith; Slater, Mariam K.: and Adler, Leonore Loeb, eds. Language. Sex and Gender: Does La Différence Make' A Difference"' New York: New York Academy of Sciences. 1979.

Suzuki, Hideo. "Gendai Nihongo ni-okeru Shuu-joshi no hataraki to sono soogo-shoosetsu nit suite" (On the function and cooccurrence of final verbs in Japanese). Kokugo (o Kokubungaku 53, no. 11: 58-70.

Suzuki, Takao, "Nihongo no jishooshi" (First person pronoun in Japanese), in Nihon Bunka to Sekai (Japanese Culture and the World). Tokyo: Koodansha, 1972.

Thorne, Barrie, and Henley, Nancy. eds. Language and Sex: Difference and Dominance. Rowley. Mass.: Newbury House, 1975.

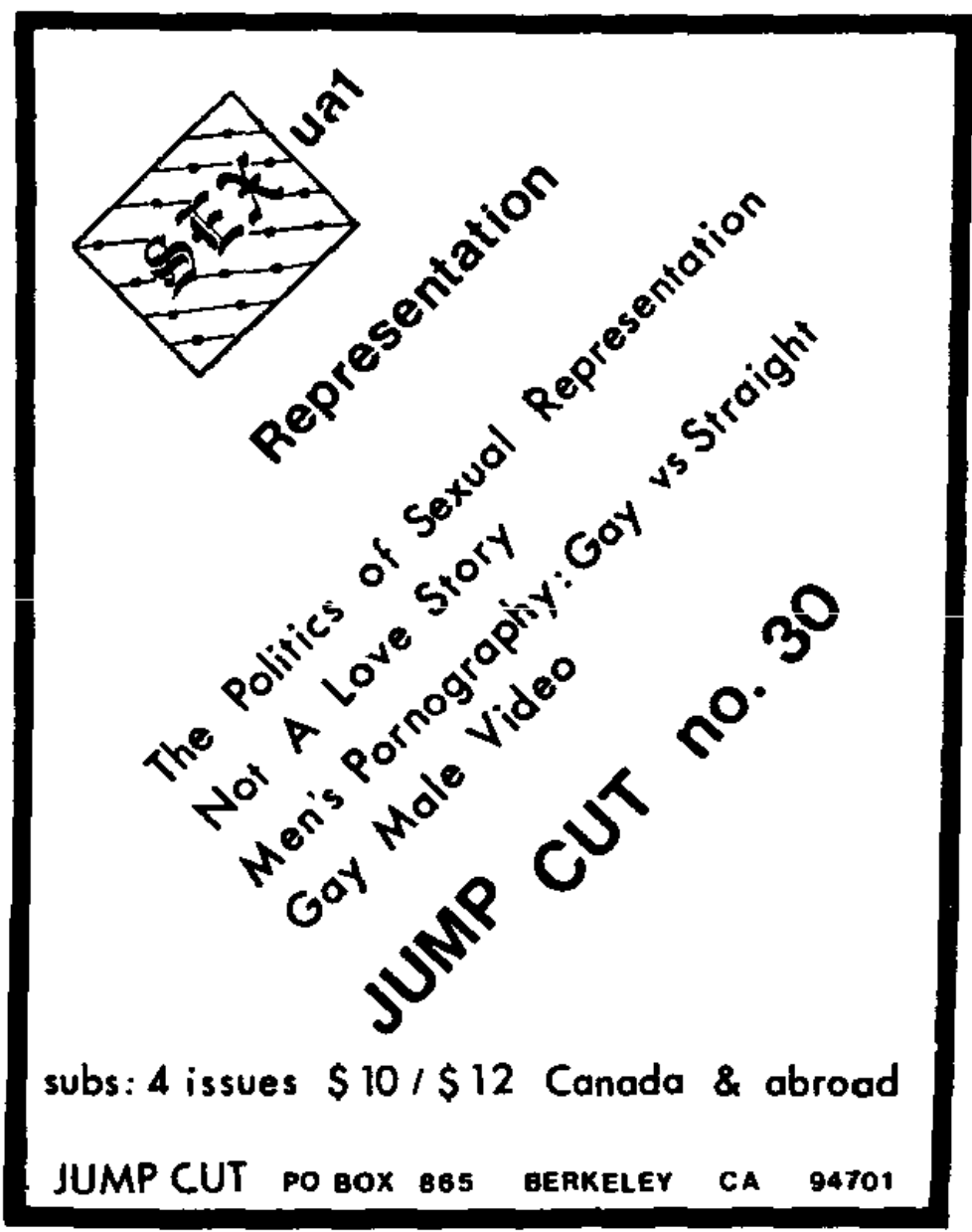

